\title{
Impact of Survival Rate and Safety Analysis of Concurrent Chemoradiotherapy in Patients with Recurrent Cervical Cancer
}

Guohua Liang*, Yajun Zhu, Qianqian Yu, Wangsheng Tian

People's Hospital of Jintan District, Changzhou 213200, Jiangsu Provinces, China

\begin{abstract}
Objective: To investigate the effect of concurrent chemoradiotherapy on the survival rate and safety of patients with recurrent cervical cancer. Methods: A total of 107 patients with recurrent cervical cancer who were treated in our hospital from March 2016 to January 2019 were retrospectively analyzed and randomly divided into the control group $(\mathrm{n}=53)$ and the observation group $(n=54)$ and treated conventionally. On this basis, the control group was treated with radiotherapy, and the observation group was treated with concurrent radiotherapy and chemotherapy. The clinical efficacy, cellular immune index, survival rate and rate of adverse reactions were compared between the two groups. Results: Compared with the total effective rate of $79.25 \%$ in the control group, the observation group was $94.44 \%$, and the difference was statistically significant $(P<0.05)$. After treatment, the levels of $\mathrm{NK}, \mathrm{CD} 3+$, and $\mathrm{CD} 4+$ in the two groups were higher than before the treatment, and the observation group was higher than the control group. The difference was statistically significant $(P<0.05)$. Compared with the adverse reaction rate of $18.87 \%$ in the control group, the observation group was $11.11 \%$, but the difference was not statistically significant $(P>0.05)$. Conclusion: Concurrent chemoradiotherapy for patients with recurrent cervical cancer has a significant effect, which not only can effectively improve the cellular immune index and the survival rate of patients, but also have high safety.
\end{abstract}

Keywords: Recurrent cervical cancer; Concurrent chemoradiotherapy; Survival rate; Cellular immune index; Incidence of adverse reactions

Publication date: November, 2019
Publication online: 10 November, 2019

*Corresponding author: Guohua Liang, lgh671206@ sina.com

\section{Introduction}

Cervical cancer is one of the common malignant tumors in gynecology. It often has asymptomatic signs in the early stage and most of the patients found the disease during physical examination. The clinical symptoms after the disease worsens are vaginal bleeding, drainage, etc., which seriously affects the life and health of patients ${ }^{[1]}$. Radiotherapy is currently a common way to treat the disease clinically. Radiation therapy of the lesion can effectively inhibit tumor growth, but it is easy to relapse, and the survival rate of patients is low. With the development of medical technology in China, concurrent chemoradiotherapy is gradually applied to the treatment of recurrent cervical cancer. Chemotherapy concurrently with radiotherapy in patients can effectively improve the treatment effect of the disease and reduce the relapse rate ${ }^{[2]}$. In view of this, this study will explore the effect of concurrent chemoradiotherapy on survival and safety of patients with recurrent cervical cancer. The report is as follows.

\section{Information and method}

\subsection{General Information}

A total of 107 patients with recurrent cervical cancer who were treated in our hospital from March 2016 to January 2019 were retrospectively analyzed and randomly divided into the control group $(\mathrm{n}=53)$ and the observation group $(\mathrm{n}=54)$. The control group was 43-74 years old, with an average age of $(58.61 \pm 4.03)$ 
years; 11 cases had pelvic wall transfer, 9 cases had bladder-rectal fossa metastasis, 8 cases had para-aortic lymph node metastasis, 12 cases had pelvic metastasis, and 13 cases had local recurrence. The observation group was 42-75 years old, with an average age of $(58.16 \pm 4.37)$ years; 10 cases had pelvic wall transfer, 8 had bladder-rectal fossa metastasis, 7 had para-aortic lymph node metastasis, 14 had pelvic metastasis, and 15 had local recurrence. The baseline data of the two groups were compared statistically, and the difference was not statistically significant $(P>0.05)$, which is comparable.

\subsection{Inclusion criteria and Exclusion criteria}

(1) Inclusion criteria: meet the diagnostic criteria for recurrent cervical cancer in Modern Oncology ${ }^{[3]}$; pathologically diagnosis of recurrent cervical cancer; complete clinical data. (2) Exclusion criteria: severe heart, liver, kidney and other important organ dysfunction; combined with other malignant tumors; abnormal blood coagulation function; mental abnormalities, unable to communicate normally.

\subsection{Methods}

Patients in both groups were given symptomatic treatment such as anti-infection, antiemetic, and diuresis during treatment.

\subsubsection{Control group}

The patients were treated with radiotherapy. The patients were treated with conventional radiation therapy on the front and rear fields of the pelvic cavity at a dose of 25-55 Gy. If a mass is found in the uterine invasion, then shrinking irradiation is performed, and the radiation dose is adjusted to a field irradiation spot dose of 10-15 Gy; if the vaginal infiltration appears a mass, the radiation dose is adjusted to $30-50 \mathrm{~Gy}$.

\subsubsection{Observation group}

The patients were given concurrent chemoradiotherapy. the plan was as follows: patients were treated with paclitaxel injection (Shanxi Pudepharma Co., Ltd., SFDA approval number H20053005, specification
$5 \mathrm{ml}: 30 \mathrm{mg}$ ). $150 \mathrm{mg} / \mathrm{m}^{2}$ paclitaxel injection was placed intravenously in a $500 \mathrm{ml}$ glucose solution. After 24 hours, the patient was treated with cisplatin injection (Jiangsu Haosen Pharma Group Co., Ltd., SFDA approval number H20040812, specification 2 $\mathrm{ml}: 10 \mathrm{mg}$ ). $70 \mathrm{mg} / \mathrm{m}^{2}$ cisplatin injection was placed intravenously in a $500 \mathrm{ml}$ glucose solution; both drugs had a cycle of 21 days. After a week of rest, the patient was treated for the next cycle, starting on the fifth day after each chemotherapy. The patients in both groups were treated continuously for 3 cycles.

\subsection{Evaluation Index}

(1)Compare the clinical efficacy of the two groups after 3 cycles of treatment. Criterion: Significantly effective: tumor disappeared and duration $>1$ month; Effective: tumor reduced volume $>50 \%$ and duration $>$ 1 Month; Ineffective: below the above criteria. The sum of the significant efficiency and the efficiency is the total effective rate. (2) Take $5 \mathrm{ml}$ of fasting venous blood in the morning before treatment and 3 months after treatment in the two groups. Centrifuge the serum to determine the cellular immunity index using flow cytometry, including $\mathrm{NK}, \mathrm{CD}^{+}$and $\mathrm{CD}^{+}$level. (3) Record the physical condition of the patients in the two groups in one year. (4) Record the occurrence of adverse reactions during the treatment in the two groups, such as PLT reduction, intestinal reactions, liver and kidney damage.

\subsection{Statistical methods}

SPSS 18.0 software was used for data processing. The measurement data and counting data were expressed as $x \pm s$ and $\mathrm{n}(\%) . T$ and $\chi^{2}$ tests were used respectively. $P<0.05$ was considered statistically significant.

\section{Result}

\subsection{Clinical effect}

Compared with the total effective rate of $79.25 \%$ in the control group, the observation group was $94.44 \%$, and the difference was statistically significant $(P<0.05)$. (Table 1$)$.

Table 1. Comparison of clinical efficacy between two groups of patients n (\%)

\begin{tabular}{ccccc}
\hline groups & significantly effective & effective & ineffective & total effective rate \\
\hline control group $(\mathrm{n}=53)$ & $23(43.40)$ & $19(35.85)$ & $11(20.75)$ & $42(79.25)$ \\
observation group $(\mathrm{n}=54)$ & $37(68.52)$ & $14(25.93)$ & $3(5.56)$ & $51(94.44)$ \\
$\chi^{2}$ & - & - & - & 5.434 \\
$P$ & - & - & - & 0.020 \\
\hline
\end{tabular}




\subsection{Immune cell index}

Before treatment, the levels of $\mathrm{NK}, \mathrm{CD}^{+}$, and $\mathrm{CD}^{+}$ were compared between the two groups, and there was no significant difference $(P>0.05)$. After treatment, the levels of $\mathrm{NK}, \mathrm{CD}^{+}$, and $\mathrm{CD}^{+}$were higher in the two groups than before treatment, and the observation group was higher than the control group. The difference was statistically significant $(P<0.05)$. (Table 2$)$.

Table 2. Comparison of immune cell index between two groups of patients $(x \pm s \%)$

\begin{tabular}{|c|c|c|c|c|}
\hline time & groups & NK & $\mathrm{CD3}^{+}$ & $\mathrm{CD}^{+}$ \\
\hline \multirow{4}{*}{ before treatment } & control group $(\mathrm{n}=53)$ & $12.78 \pm 3.47$ & $58.81 \pm 5.30$ & $25.91 \pm 4.31$ \\
\hline & observation group $(n=54)$ & $13.06 \pm 3.59$ & $59.03 \pm 5.16$ & $26.08 \pm 4.10$ \\
\hline & $t$ & 0.410 & 0.218 & 0.209 \\
\hline & $P$ & 0.683 & 0.828 & 0.835 \\
\hline \multirow{4}{*}{ after treatment } & control group $(\mathrm{n}=53)$ & $16.28 \pm 4.01^{\mathrm{a}}$ & $63.07 \pm 6.23^{\mathrm{a}}$ & $29.20 \pm 4.26^{\mathrm{a}}$ \\
\hline & observation group $(\mathrm{n}=54)$ & $20.14 \pm 4.02^{\mathrm{a}}$ & $70.14 \pm 6.15^{\mathrm{a}}$ & $34.51 \pm 4.23^{\mathrm{a}}$ \\
\hline & $t$ & 4.972 & 5.907 & 6.470 \\
\hline & $P$ & 0.001 & 0.000 & 0.000 \\
\hline
\end{tabular}

Note: Compared with the same group before treatment, ${ }^{\mathrm{a}} P<0.05$.

\subsection{Survival rate}

40 patients in the control group survived, and the overall survival rate was $75.47 \%$; The survival rate of 49 patients in the observation group was $90.74 \%$. The difference between groups was statistically significant $\left(\chi^{2}=4.457, \mathrm{P}=0.035\right)$.

\subsection{Incidence of adverse reactions}

The number of PLT reduction, intestinal reactions, and liver and kidney injury in the control group was 3, 5, and 2 respectively, with a total incidence of $18.87 \%$. In the observation group, there were 1,3 and 2 cases respectively, with a total incidence of $11.11 \%$. There was no significant difference between the groups $\left(\chi^{2}=1.266, P=0.261\right)$.

\section{Discussion}

Cervical cancer is a common reproductive tract cancer disease in gynecology, and it is the "second biggest killer" that threatens women's health, second only to breast cancer. Its etiology is unclear, and it is mostly considered to be related to premature sexual behavior, multiple sexual partners, smoking, environmental pollution, etc. It has the characteristics of high recurrence rate and low cure rate. If not treated in time, it may cause ureteral obstruction, hydronephrosis, uremia, etc. In the later stage, there will be systemic organic lesions that are life-threatening ${ }^{[4]}$. At present, the clinical treatment of this disease is usually performed by surgery, chemotherapy, radiotherapy, etc., but some of the effects are not satisfactory. Therefore, finding a high-quality treatment plan is of great significance to improve clinical efficacy and survival rate of the patients.

Radiotherapy is a way to treat tumor by radiation, which belongs to local treatment. Although it has a certain effect on cervical cancer, it is not satisfactory. Mainly because of the hypoxic cells in the tumor center, it is not sensitive to radiotherapy, so this method cannot completely suppress tumor cells. However, increasing the radiotherapy dose not only has a strong toxic and complex effect, but also cannot be afforded by most patients. so the therapeutic effect is limited, which is not conducive to clinical promotion ${ }^{[5-6]}$. The results of this study show that the clinical efficacy, immune index, and survival rate of the observation group are better than those of the control group, and the incidence of adverse reactions is slightly lower, indicating that patients with recurrent cervical cancer have the exact effect of concurrent chemoradiotherapy. The reasons are as follows: Paclitaxel and cisplatin are first-line chemotherapy drugs. Paclitaxel can inhibit the mitotic process of cancer cells and promote the autonomous apoptosis of cancer cells. Cisplatin is a platinum-containing anticancer drug that can bind to cancer cell DNA and cause cross-linking, thus destroying the function of DNA and inhibiting mitosis of cells to achieve the purpose of anti-tumor; Combining them with radiotherapy in combination with patients can change the permeability of tumor cells in patients' lesions through radiotherapy, promote 
the rapid effect of chemotherapy drugs in the body, and play a role in inhibiting tumor growth; In addition, the drug used in chemotherapy can also increase the sensitivity of tumor cells to radiation therapy. During the simultaneous treatment process, it can effectively shorten the onset time of drugs and improve the clinical treatment effect ${ }^{[7-9]}$. It can be seen that the simultaneous application of two methods of radiotherapy and chemotherapy can make use of their different mechanisms to play a role in suppressing tumor growth, which is worthy of clinical promotion and application.

In summary, the use of concurrent chemoradiotherapy in patients with recurrent cervical cancer has a significant effect, which can effectively improve the cellular immune index of patients, improve the survival rate, and have high safety.

\section{References}

[1] Wang WL, Qu ZY, Zhao GQ. Clinical efficacy analysis of concurrent chemoradiotherapy for recurrent cervical cancer[J]. Chinese Journal of Cancer Prevention and Treatment, 2019, 26(8): 570-572.

[2] Zhang SJ, Zhou YG, Keyoumu A. Decoction of nourishing healthy qi and eliminating blood stasis combined with concurrent chemoradiotherapy in the treatment of patients with cervical cancer and its effect on the body's immune function [J].
Modern Journal of Integrated Traditional Chinese and Western Medicine, 2017, 26(26): 2921-2923.

[3] Tang ZY. Modern Oncology [M]. Shanghai: Fudan University Press, 2011: 569-571.

[4] Li J, Qu JR, Zhang HK, et al. Evaluation of the value of simultaneous radiotherapy and chemotherapy for locally advanced cervical cancer by intra-voxel irrelevant motion imaging [J]. Chinese Journal of Radiology, 2018, 52(3): 192198.

[5] Yan YY, Pan DF, Cao FJ. Application of compound Banzhi Capsule combined with concurrent chemoradiotherapy in cervical cancer [J]. Journal of International Oncology, 2016, 43(11): 826-828.

[6] An JS, Du XY, Zhang F, et al. Effect of radiotherapy on ovarian function in patients with radical cervical cancer surgery [J]. Chinese Journal of Radiation Oncology, 2019, 28(10): 753-757.

[7] Ji J, Yang YQ, Xu XT, et al. Study on cancer-related fatigue and anxiety and depression in patients with cervical cancer undergoing concurrent chemoradiotherapy [J]. Journal of Nursing Management, 2019, 19(8): 573-576.

[8] Cai SN, Ha K, Zhang YX, et al. Influential factors of prognosis in patients with advanced cervical cancer undergoing concurrent chemoradiotherapy [J]. Journal of PLA Medical College, 2016, 37(11): 1136-1141.

[9] Chen QM, Li JQ. Clinical study of Xihuang pills combined with concurrent chemoradiotherapy for local cervical cancer [J]. World Journal of Traditional Chinese Medicine, 2016, 11(7): 1215-1217. 